\title{
Fantastic Features and Where to Find Them: Detecting Cognitive Impairment with a Subsequence Classification Guided Approach
}

\author{
Benjamin Eyre \\ Winterlight Labs \\ Toronto, Canada \\ Aparna Balagopalan \\ Winterlight Labs \\ Toronto, Canada \\ Jekaterina Novikova \\ Winterlight Labs \\ Toronto, Canada \\ \{benjamin, aparna, jekaterina\}@winterlightlabs.com
}

\begin{abstract}
Despite the widely reported success of embedding-based machine learning methods on natural language processing tasks, the use of more easily interpreted engineered features remains common in fields such as cognitive impairment (CI) detection. Manually engineering features from noisy text is time and resource consuming, and can potentially result in features that do not enhance model performance. To combat this, we describe a new approach to feature engineering that leverages sequential machine learning models and domain knowledge to predict which features help enhance performance. We provide a concrete example of this method on a standard data set of CI speech and demonstrate that CI classification accuracy improves by $2.3 \%$ over a strong baseline when using features produced by this method. This demonstration provides an example of how this method can be used to assist classification in fields where interpretability is important, such as health care.
\end{abstract}

\section{Introduction}

In recent years, word and sentence embeddingbased methods have had a significant impact on the field of NLP (Devlin et al., 2019; Mikolov et al., 2013; Pennington et al., 2014; Di Palo and Parde, 2019). These approaches stand as an alternative to classical feature engineering approaches, where carefully crafted features, such as word length or part of speech tag, are extracted from text and used as input. Despite the promise of embeddingbased methods, there are still several advantages to feature engineering. Most notably, using embeddings as input can lead to issues with interpretability (Heimerl and Gleicher, 2018; Hooker et al., 2019; Kindermans et al., 2017), which is especially important in a healthcare domain (Balagopalan et al., 2020). Meanwhile, feature engineering approaches directly lend themselves to easily inter- pretable models (Ribeiro et al., 2016). As such, feature engineering remains an important practice for fields such as health care, where interpretability is imperative. An extensive body of work has been produced where ML methods and engineered features have been applied to cognitive impairment (CI) detection (Balagopalan et al., 2018; Karlekar et al., 2018; Zhu et al., 2019).

In this work, we present a new feature engineering method that is guided by classifying subsets of a pause-centred speech sequence (subsequences), and inspired by literature suggesting that CI could be indicated by the words that subjects pause before (Calley et al., 2010; Mack et al., 2013; Seifart et al., 2018). This approach aims to extract pauserelated information while minimizing the noise added from unrelated factors. This method generates interpretable and effective features, potentially saving time and resources spent on excess feature engineering. We validate this method by presenting a 2.3\% accuracy increase over a strong baseline on CI vs healthy (HC) classification, matching the state of the art (Hernández-Domínguez et al., 2018).

In summary, our major contributions are:

- A method of classifying speech using only a token of interest and a small context around it, i.e. subsequence classification (Sec. 3.3).

- A novel feature engineering approach guided by subseqence classification (Sec. 4).

- Validating this approach by showing that it aids in achieving classification results comparable to the state of the art (Sec. 5.2).

\section{Related work}

Several authors report increases in CI detection performance by extracting acoustic features such as filled and unfilled pause counts, as well as average pause duration (Tóth et al., 2015, 2018; Pistono 


\begin{tabular}{cccc}
\hline Data Subset & HC & CI & Total \\
\hline \hline DB (transcripts) & $229(42 \%)$ & $321(58 \%)$ & 550 \\
\hline DB-C1 & $317(33 \%)$ & $645(67 \%)$ & 962 \\
DB-C2 & $511(35 \%)$ & $963(65 \%)$ & 1,474 \\
DB-C3 & $529(35 \%)$ & $980(65 \%)$ & 1,509 \\
DB-Utt & $755(42 \%)$ & $1,059(58 \%)$ & 1,814 \\
\hline
\end{tabular}

Table 1: Overview of the number of samples (subsequences or transcripts) in different subsets of DB.

et al., 2016). However, we believe further performance increases can be achieved if we focus not only on the pauses themselves, but also the linguistic context in which the pauses occur in speech.

Recently, Hernández-Domínguez et al. (2018) achieved an accuracy of $78 \%$ when classifying Dementiabank (Sec. 3.1) transcripts as CI or HC using an extended set of lexical features, which is to the best of our knowledge the state of the art (SOTA). We use their recorded performance as a benchmark when validating our approach.

Several authors have reported performance gains by using subsequences to aid with classification. These authors use subsequences only as a means to process full sequences (Phan et al., 2017), or they use the presence of common subsequences as a feature for longer text sequences (Iglesias et al., 2007; Kumar et al., 2005). To the best of our knowledge, no prior work describes using subsequence classification to guide feature engineering.

\section{Experimental Method}

In this section, we define the data sets and methodology used in our experimental framework.

\subsection{Data Sets}

Dementiabank (DB): Dementiabank ${ }^{1}$ is a large public data set of pathological speech (Becker et al., 1994), containing audio files and transcripts of participants describing the 'Cookie Theft' image. Transcripts are created manually by trained transcriptionists following the CHAT protocol (MacWhinney, 2014). Out of the 286 participants, 193 are diagnosed with some form of $\mathrm{CI}(\mathrm{CI} ; \mathrm{N}=$ 321 transcripts) and 93 are healthy controls (HC; N $=229$ transcripts). Transcripts receive a CI or HC label corresponding to whether the participant who produced the transcribed speech was cognitively impaired or not.

Subsequence-based Data Subsets: To conduct subsequence classification, we extract subsequences of varying length from each transcript.

\footnotetext{
${ }^{1}$ https://dementia.talkbank.org
}

\begin{tabular}{|c|c|c|c|c|c|c|}
\hline D3 & D2 & D1 & & D1 & D2 & D3 \\
\hline $\begin{array}{l}\text { word } \\
\text { token }\end{array}$ & $\begin{array}{l}\text { word } \\
\text { token }\end{array}$ & $\begin{array}{l}\text { word } \\
\text { token }\end{array}$ & pause & $\begin{array}{l}\text { word } \\
\text { token }\end{array}$ & $\begin{array}{l}\text { word } \\
\text { token }\end{array}$ & $\begin{array}{l}\text { word } \\
\text { token }\end{array}$ \\
\hline & & C2 L & C1 & 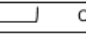 & & \\
\hline
\end{tabular}

Figure 1: Visualization of the difference between contexts and distances in a pause-focussed subsequence.

For each transcript in DB, each utterance containing a pause was extracted and labelled as positive if the sample contains CI speech, or negative otherwise. Subsequences were extracted from these utterances by taking the first one, two, or three speech tokens before and after each pause. ${ }^{2}$ We created three data subsets by including subsequences of at most one, two, or three tokens around the pause: Context 1 (DB-C1), Context 2 (DB-C2), and Context 3 (DB-C3), respectively. We also included one data subset including full utterances that include pauses, DB-Utt (Tab.1). Identical subsequences found in both classes were removed. Furthermore, subsequences extracted from $\mathrm{HC}$ transcripts are labeled as $\mathrm{HC}$, and subsequences extracted from CI transcripts are labeled as CI. We refer to the tokens that are next to the pause as Distance 1 (D1), the tokens that are one token away from the pause as Distance 2 (D2), and the tokens that are two tokens away from the pause as Distance 3 (D3). The differences between context and distance are shown in Fig.1. For example, for the pause sequence "The boy is *uh* stealing a cookie", only the tokens "boy" and " $a$ " would be considered the Distance 2 tokens for this sequence, while the tokens "boy", "is", "* $u h^{*}$ ", "stealing", and " $a$ " would be considered the Context 2 tokens for this sequence.

\subsection{Feature Extraction}

In this section, we describe how features are extracted on the transcript-level (for transcript classification) and on the token-level (for subsequence classification).

Transcript-Level Features: We extract over 500 linguistic and acoustic features from each transcript, such as part of speech counts and average word length (App.A). These features, referred to as the Original feature set, are used to provide a baseline to benchmark transcript-level classification performance. We also use the Original feature

\footnotetext{
${ }^{2}$ If there were less than two or three tokens before or after a pause, the largest possible sequence of tokens was extracted.
} 
set as a base that we extend with newly engineered transcript-level features (Sec. 4). To produce an additional baseline, we perform feature selection on the Original feature set, and found $k=85$ features led to the greatest performance.

Token-Level Features: In order to conduct subsequence classification, we extract features on the token-level for each of the subsequence-based data subsets. Of the Original feature set, we select a subset of features that have a clear token-level analogue (App.A). For instance, the transcript-level feature of average word length has the token-level analogue of individual word length. After feature extraction, each token is represented by a 23dimensional input vector. Consequently, each subsequence in the DB-C1, DB-C2, DB-C3, and DBUtt data subsets is represented as a $T$ by 23 matrix, where $T$ is the length of the sequence in tokens.

\subsection{Classification}

In this section, we describe the methodology used for subsequence and transcript classification. Subsequence classification is used to guide the engineering of new features, while transcript classification validates the new features' effectiveness.

Subsequence Classification: Our subsequence classification experiment involves performing 5fold cross validation with each of the DB-C1, DBC2, DB-C3, and DB-Utt data subsets. Subsequences are classified as either $\mathrm{HC}$ or $\mathrm{CI}$. We conduct classification using GRU-based (Cho et al., 2014) models with an attention mechanism designed for document classification (Yang et al., 2016), with model parameters tuned for each of the data subsets. We report accuracy for M-C1, the model that achieved the highest accuracy on DB$\mathrm{C} 1, \mathrm{M}-\mathrm{C} 2$, the model that achieved the highest accuracy on DB-C2, M-C3, the model that achieved the highest accuracy on DB-C3, and M-Utt, the model that achieved the highest accuracy on DBUtt (additional training details provided in App.B). Transcript Classification: We evaluate the efficacy of our feature engineering approach (Sec.4) by performing transcript-level 10 -fold cross validation with a variety of feature sets. Transcripts are classified as either HC or CI. We use the Original feature set, as well as the top 85 of the Original features, based on their ANOVA F-values, as baselines. Additionally, we extend the Original feature set with the $k$ best features, based on ANOVA F-values, from each of the feature sets generated using our novel feature engineering approach (Sec. 4 ), separately. $k$ is optimized for accuracy for each extending feature set separately. To classify DB transcripts, we use 5 ML models: an SVM, a gradient boosting ensemble, a 2-layer neural network $(\mathrm{NN})$, a random forest, and an ensemble of the previous four models (Ens). We report the accuracy (Acc), precision (Prec), sensitivity (Sens), and specificity (Spec) for the model that achieved the greatest cross validated accuracy for each feature set, separately (training details provided in App.B).

\section{Proposed Feature Engineering Approach}

Our approach to engineering new transcript-level features involves three major steps:

1) Subsequences of varying length centred around a token of interest, in our case a pause, must be extracted from each of the input transcripts and grouped into subsets based on maximum length. Each of the tokens in these subsequences must have token-level features extracted. The tokenlevel features, as well as the central token, should be chosen based on in-domain knowledge.

2) A sequential ML model must be cross validated on each of the subsequence data subsets from the previous step in a subsequence classification experiment. Here, we are specifically attempting to exploit the ability for sequential machine learning models to uncover patterns in sequential data. The mean cross validated accuracy on each of these length-based data subsets should be used as an indicator of how much distinguishing information can be extracted from tokens within the specified range of the pause.

3) Based on the recorded cross validated accuracies from the previous step, transcript-level aggregations of the token-level features must be created at various distances from the pause. We propose two methods of aggregating token-level features (DB-specific details provided in App.A):

- Continuous features can be aggregated simply by taking the average of a feature across each of the tokens. An example of this would be calculating the average word length for each of the tokens found at a specified distance from a pause.

- Categorical features can be aggregated using counts or ratios, such as the number of nouns occurring at a specified distance from a pause.

These transcript-level aggregates should only be extracted for the distances that produced the great- 


\begin{tabular}{cccccc}
\hline Feature Set & Model & Acc & Prec & Sens & Spec \\
\hline \hline Original & Ens & $74.77 \pm 0.6^{*}$ & $82.08 \pm 0.4^{*}$ & $73.1 \pm 0.5^{*}$ & $79.74 \pm 0.4^{*}$ \\
Original w/ feat.sel. & Ens & $75.18 \pm 1.4$ & $83.37 \pm 1.2$ & $72.21 \pm 1.3^{*}$ & $81.67 \pm 1.6$ \\
\hline Original + F-D1 & NN & $74.41 \pm 1.9^{*}$ & $78.59 \pm 1.1^{*}$ & $\mathbf{7 7 . 1 5} \pm \mathbf{3 . 3}$ & $72.63 \pm 1.9^{*}$ \\
Original + F-D2 & Ens & $\mathbf{7 7 . 0 9} \pm \mathbf{1 . 0}$ & $\mathbf{8 4 . 4 0} \pm \mathbf{0 . 8}$ & $75.21 \pm 1.0$ & $82.32 \pm 0.9$ \\
Original + F-D3 & Ens & $76.05 \pm 0.8$ & $84.02 \pm 0.9$ & $71.92 \pm 0.7^{*}$ & $\mathbf{8 3 . 3 3} \pm \mathbf{1 . 3}$ \\
Original + F-C2 & NN & $75.14 \pm 1.4$ & $79.85 \pm 1.5^{*}$ & $76.93 \pm 0.9^{*}$ & $74.02 \pm 2.5^{*}$ \\
Original + F-C3 & Ens & $74.82 \pm 1.2^{*}$ & $83.68 \pm 1.2$ & $70.62 \pm 1.9^{*}$ & $82.63 \pm 1.4$ \\
\hline
\end{tabular}

Table 2: Transcript classification performance for each feature set's best performing classification model, averaged across four random seeds. Bold indicates best performance, and * indicates significance $(p<0.05)$ when compared to the model using F-D2 features.

\begin{tabular}{ccccc}
\hline Model & M-C1 & M-C2 & M-C3 & M-Utt \\
\hline Accuracy & $59.6 \pm 2.6$ & $\mathbf{6 0 . 7} \pm \mathbf{2 . 5}$ & $59.8 \pm 0.9$ & $60.3 \pm 1.0$
\end{tabular}

Table 3: Subsequence classification performance. Accuracy is averaged across four random seeds.

est cross validated accuracy during subsequence classification, as the subsequence classification performance indicates that the features found in that range are the most distinguishing. For instance, if subsequences of up to two tokens around a pause produced the most accurate subsequence classifier, transcript-level aggregates should only be extracted for tokens at the D1 and D2 positions in reference to the pause, and not the $\mathrm{D} 3$ position.

To validate this method, we create five transcriptlevel feature sets: features aggregated from tokens at the $\mathrm{D} 1$ position in reference to a pause $(F-D 1)$, features aggregated from the $\mathrm{D} 2$ position $(F-D 2)$, features aggregated from the $\mathrm{D} 3$ position $(F-D 3)$, the combination of F-D1, F-D2, and F-D3 ( $F-C 3)$, and the combination of F-D1 and F-D2 $(F-C 2)$.

\section{Results}

In this section, we report the results for the subsequence and transcript classification experiments.

\subsection{Subsequence Classification}

After averaging across four random seeds, M-C2 was able to achieve an accuracy of $60.7 \%$, higher than M-C1, M-C3, or M-Utt (Tab.3). This leads us to the conclusion that using features from the two tokens preceding and succeeding a pause could enhance transcript-level classification performance.

\subsection{Transcript Classification}

We create the F-D1, F-D2, and F-C2 aggregate feature sets, as the highest subsequence classification accuracy was achieved by a model trained on DBC2. Additionally, in order to validate our claims, we create F-D3 and F-C3. The highest accuracy of $77.09 \%$ on transcript classification is achieved by an ensemble model that used the Original + F-D2 feature set (Tab. 2).

Using one of the four random seeds used to produce the average performance metrics presented in Tab. 2, the model using F-D2 features was able to achieve an accuracy of $78.36 \%$, the same as the single-seed SOTA accuracy of $78 \%$ (HernándezDomínguez et al., 2018).

\section{Discussion}

As shown in Tab. 3 and Tab. 2, features from tokens within 2 tokens of a pause were the most effective in enhancing both subsequence and transcript classification. To determine how these two tasks are connected, we conduct a statistical analysis on the token-level and transcript-level features. Two sided t-tests between features extracted from tokens found at D1, D2, and D3 from different classes show similar patterns for features that are significantly different between classes for both the token and transcript-level. Larger concentrations of distinguishing features are found at D1 and D2 than at D3. This could explain the effectiveness of features from the D2 position in both tasks (Tab. 4).

However, this pattern congruity does not explain why F-D3 features on their own are more effective than F-D1 features on their own. The trend that both the F-D3 and F-C3 feature sets produced greater transcript-level accuracy than the F-D1 feature set, and lower transcript-level accuracy than the F-D2 and F-C2 feature sets, is the same as the trend for the subsequence classification results reported in Tab. 3. This indicates that subsequence classification may be able to provide better insight into potential transcript classification performance than traditional statistical testing.

It is important to consider the implications of producing a model with the F-D2 feature set that 


\begin{tabular}{ccc}
\hline Distance & Token-Level & Transcript-Level \\
\hline \hline D1 & 18 & 12 \\
D2 & 21 & 12 \\
D3 & 7 & 6 \\
\hline
\end{tabular}

Table 4: Number of features that are significantly different between classes according to two sided t-tests for each distance.

achieved significantly higher accuracy than the most accurate model produced with the F-C 3 feature set. As described in Section 3.3, we perform feature selection using ANOVA F-values for each of the aggregate feature sets. Since F-D2 is a subset of F-C3, this implies that this more traditional feature selection method did not select a group of features from $\mathrm{F}-\mathrm{C} 3$ that was more effective than the features from F-D2, even though it was able to select any and all of the features in F-D2. This serves as a testament to our feature engineering method, as it demonstrates that even popular feature selection methods are not able to completely remove the negative effects of engineering an excessive amount of ineffective features.

Following several other works that used the DB data set (Hernández-Domínguez et al., 2018; PouProm and Rudzicz, 2018; Sarawgi et al., 2020), all of our experiments are conducted with K-fold cross validation. While the small size of the DB data set helps to justify this as a validation procedure, optimizing a cross validated performance metric (accuracy, F1, etc.) may lead results using $\mathrm{K}$-fold cross validation to be an overestimate of generalization performance.

DB-C2 produced a more accurate subsequence classifier than any other data subset of DB. This suggests that the class distinguishing signal from the pause is strongest within a two token radius around the pause. Beyond that radius, the signal may be obstructed by noise from other patterns in speech. However, in different data sets, a different subsequence length may present the strongest, least noisy signal. New aggregate features should be created for tokens within whichever range produces the best subsequence classification performance.

However, our results do indicate that there is a strong link between how well features from certain token positions contribute to both subsequence and transcript classification. This may relate to the effect of noise on those token positions, which we use subsequence classification to identify.

\section{Conclusion and Future Work}

In this work, we present two principle contributions. First, we describe a novel method for speech classification - subsequence classification - in which speech is modelled as a token of interest, such as a pause, along with surrounding tokens of context. Secondly, we demonstrate how subsequence classification can be used to engineer features that extract distinguishing information while minimizing added noise, and consequently match SOTA performance on a standard data set of CI speech.

Future work should be done to understand why certain context lengths are more conducive for subsequence classification than others, and when that performance can transfer to effective transcriptlevel classification. Finally, additional work should be done to develop techniques for finding tokens of interest, such as pauses, that can be exploited using our feature engineering technique. 


\section{References}

Aparna Balagopalan, Benjamin Eyre, and Jekaterina Novikova. 2020. To BERT or Not To BERT: Comparing Speech and Language-based Approaches for Alzheimer's Disease Detection. In Proceedings of INTERSPEECH.

Aparna Balagopalan, Jekaterina Novikova, Frank Rudzicz, and Marzyeh Ghassemi. 2018. The effect of heterogeneous data for alzheimer's disease detection from speech. In Proceedings of the MLAH Workshop at NeurIPS.

James T Becker, François Boiler, Oscar L Lopez, Judith Saxton, and Karen L McGonigle. 1994. The natural history of Alzheimer's disease: description of study cohort and accuracy of diagnosis. Archives of Neurology, 51(6):585-594.

Marc Brysbaert, Amy Beth Warriner, and Victor Kuperman. 2014. Concreteness ratings for 40 thousand generally known english word lemmas. Behavior Research Methods, 46(3):904-911.

Clifford S Calley, Gail D Tillman, Kyle Womack, Patricia Moore, John Hart Jr, and Michael A Kraut. 2010. Subjective report of word-finding and memory deficits in normal aging and dementia. Cognitive and behavioral neurology: official journal of the Society for Behavioral and Cognitive Neurology, 23(3):185.

Nitesh V Chawla, Kevin W Bowyer, Lawrence O Hall, and W Philip Kegelmeyer. 2002. Smote: synthetic minority over-sampling technique. Journal of artificial intelligence research, 16:321-357.

Kyunghyun Cho, Bart Van Merriënboer, Caglar Gulcehre, Dzmitry Bahdanau, Fethi Bougares, Holger Schwenk, and Yoshua Bengio. 2014. Learning phrase representations using rnn encoder-decoder for statistical machine translation. arXiv preprint arXiv:1406.1078.

Mark Davies. 2009. The 385+ million word corpus of contemporary american english (1990-2008+): Design, architecture, and linguistic insights. International journal of corpus linguistics, 14(2):159-190.

Jacob Devlin, Ming-Wei Chang, Kenton Lee, and Kristina Toutanova. 2019. Bert: Pre-training of deep bidirectional transformers for language understanding. In Proceedings of the 2019 Conference of the North American Chapter of the Association for Computational Linguistics: Human Language Technologies, Volume 1 (Long and Short Papers), pages 4171-4186.

Flavio Di Palo and Natalie Parde. 2019. Enriching neural models with targeted features for dementia detection. arXiv preprint arXiv:1906.05483.

Kathleen C Fraser, Jed A Meltzer, and Frank Rudzicz. 2016. Linguistic features identify alzheimer's disease in narrative speech. Journal of Alzheimer's Disease, 49(2):407-422.
Florian Heimerl and Michael Gleicher. 2018. Interactive analysis of word vector embeddings. In Computer Graphics Forum, volume 37, pages 253-265. Wiley Online Library.

Laura Hernández-Domínguez, Sylvie Ratté, Gerardo Sierra-Martínez, and Andrés Roche-Bergua. 2018. Computer-based evaluation of alzheimer's disease and mild cognitive impairment patients during a picture description task. Alzheimer's \& Dementia: Diagnosis, Assessment \& Disease Monitoring, 10:260268.

Matthew Honnibal and Ines Montani. 2017. spaCy 2: Natural language understanding with Bloom embeddings, convolutional neural networks and incremental parsing. To appear.

Sara Hooker, Dumitru Erhan, Pieter-Jan Kindermans, and Been Kim. 2019. A benchmark for interpretability methods in deep neural networks. In Advances in Neural Information Processing Systems, pages 9737-9748.

José Antonio Iglesias, Agapito Ledezma, and Araceli Sanchis. 2007. Sequence classification using statistical pattern recognition. In International Symposium on Intelligent Data Analysis, pages 207-218. Springer.

Sweta Karlekar, Tong Niu, and Mohit Bansal. 2018. Detecting linguistic characteristics of alzheimer's dementia by interpreting neural models. In Proceedings of NAACL-HLT, pages 701-707.

Pieter-Jan Kindermans, Sara Hooker, Julius Adebayo, Maximilian Alber, Kristof T Schütt, Sven Dähne, Dumitru Erhan, and Been Kim. 2017. The (un) reliability of saliency methods. arXiv preprint arXiv:1711.00867.

N Pradeep Kumar, M Venkateswara Rao, P Radha Krishna, and Raju S Bapi. 2005. Using sub-sequence information with knn for classification of sequential data. In International Conference on Distributed Computing and Internet Technology, pages 536-546. Springer.

Victor Kuperman, Hans Stadthagen-Gonzalez, and Marc Brysbaert. 2012. Age-of-acquisition ratings for 30,000 english words. Behavior research methods, 44(4):978-990.

Jennifer E Mack, Aya Meltzer-Asscher, Sarah D. Chandler, Sandra Weintraub, Marek Marsel Mesulam, and Cynthia K Thompson. 2013. Word-finding pauses in primary progressive aphasia (ppa): Effects of lexical category. Procedia - Social and Behavioral Sciences, 94:129-130.

Brian MacWhinney. 2014. The CHILDES project: Tools for analyzing talk, Volume I: Transcription format and programs. Psychology Press. 
Tomas Mikolov, Kai Chen, Greg Corrado, and Jeffrey Dean. 2013. Efficient estimation of word representations in vector space. arXiv preprint arXiv:1301.3781.

Natalia B Mota, Nivaldo AP Vasconcelos, Nathalia Lemos, Ana C Pieretti, Osame Kinouchi, Guillermo A Cecchi, Mauro Copelli, and Sidarta Ribeiro. 2012. Speech graphs provide a quantitative measure of thought disorder in psychosis. PloS one, 7(4):e34928.

Adam Paszke, Sam Gross, Francisco Massa, Adam Lerer, James Bradbury, Gregory Chanan, Trevor Killeen, Zeming Lin, Natalia Gimelshein, Luca Antiga, Alban Desmaison, Andreas Kopf, Edward Yang, Zachary DeVito, Martin Raison, Alykhan Tejani, Sasank Chilamkurthy, Benoit Steiner, Lu Fang, Junjie Bai, and Soumith Chintala. 2019. Pytorch: An imperative style, high-performance deep learning library. In $\mathrm{H}$. Wallach, H. Larochelle, A. Beygelzimer, F. d'Alché-Buc, E. Fox, and R. Garnett, editors, Advances in Neural Information Processing Systems 32, pages 8024-8035. Curran Associates, Inc.

Fabian Pedregosa, Gaël Varoquaux, Alexandre Gramfort, Vincent Michel, Bertrand Thirion, Olivier Grisel, Mathieu Blondel, Peter Prettenhofer, Ron Weiss, Vincent Dubourg, et al. 2011. Scikit-learn: Machine learning in python. the Journal of machine Learning research, 12:2825-2830.

Jeffrey Pennington, Richard Socher, and Christopher Manning. 2014. Glove: Global vectors for word representation. In Proceedings of the 2014 Conference on Empirical Methods in Natural Language Processing (EMNLP), pages 1532-1543, Doha, Qatar. Association for Computational Linguistics.

Huy Phan, Philipp Koch, Fabrice Katzberg, Marco Maass, Radoslaw Mazur, and Alfred Mertins. 2017. Audio scene classification with deep recurrent neural networks. arXiv preprint arXiv:1703.04770.

Aurélie Pistono, Mélanie Jucla, Emmanuel J Barbeau, Laure Saint-Aubert, Béatrice Lemesle, Benjamin Calvet, Barbara Köpke, Michèle Puel, and Jérémie Pariente. 2016. Pauses during autobiographical discourse reflect episodic memory processes in early alzheimer's disease. Journal of Alzheimer's Disease, 50(3):687-698.

Chloé Pou-Prom and Frank Rudzicz. 2018. Learning multiview embeddings for assessing dementia. In Proceedings of the 2018 Conference on Empirical Methods in Natural Language Processing, pages 2812-2817.

Marco Tulio Ribeiro, Sameer Singh, and Carlos Guestrin. 2016. Why should i trust you?: Explaining the predictions of any classifier. In Proceedings of the 22nd ACM SIGKDD international conference on knowledge discovery and data mining, pages 1135-1144. ACM.
Utkarsh Sarawgi, Wazeer Zulfikar, Nouran Soliman, and Pattie Maes. 2020. Multimodal inductive transfer learning for detection of alzheimer's dementia and its severity. arXiv preprint arXiv:2009.00700.

Frank Seifart, Jan Strunk, Swintha Danielsen, Iren Hartmann, Brigitte Pakendorf, Søren Wichmann, Alena Witzlack-Makarevich, Nivja H de Jong, and Balthasar Bickel. 2018. Nouns slow down speech across structurally and culturally diverse languages. Proceedings of the National Academy of Sciences, 115(22):5720-5725.

Hans Stadthagen-Gonzalez and Colin J. Davis. 2006. The bristol norms for age of acquisition, imageability, and familiarity. Behavior Research Methods, 38(4):598-605.

Laszló Tóth, Gábor Gosztolya, Veronika Vincze, Ildikó Hoffmann, Gréta Szatlóczki, Edit Biró, Fruzsina Zsura, Magdolna Pákáski, and János Kálmán. 2015. Automatic detection of mild cognitive impairment from spontaneous speech using asr. In Sixteenth Annual Conference of the International Speech Communication Association.

László Tóth, Ildikó Hoffmann, Gábor Gosztolya, Veronika Vincze, Gréta Szatlóczki, Zoltán Bánréti, Magdolna Pákáski, and János Kálmán. 2018. A speech recognition-based solution for the automatic detection of mild cognitive impairment from spontaneous speech. Current Alzheimer Research, 15(2):130-138.

Amy Beth Warriner, Victor Kuperman, and Marc Brysbaert. 2013. Norms of valence, arousal, and dominance for 13,915 english lemmas. Behavior Research Methods, 45(4):1191-1207.

Zichao Yang, Diyi Yang, Chris Dyer, Xiodong He, Alex Smola, and Hovy. 2016. Hierarchical attention networks for document classification. In Proceedings of NAACL-HLT, pages 1480-1489.

Zining Zhu, Jekaterina Novikova, and Frank Rudzicz. 2019. Detecting cognitive impairments by agreeing on interpretations of linguistic features. In Proceedings of the 2019 Conference of the North American Chapter of the Association for Computational Linguistics: Human Language Technologies, Volume 1 (Long and Short Papers), pages 1431-1441. 